\title{
SUBDOMINANT EIGENVALUES FOR STOCHASTIC MATRICES WITH GIVEN COLUMN SUMS*
}

\author{
STEVE KIRKLAND ${ }^{\dagger}$
}

\begin{abstract}
For any stochastic matrix $A$ of order $n$, denote its eigenvalues as $\lambda_{1}(A), \ldots, \lambda_{n}(A)$, ordered so that $1=\left|\lambda_{1}(A)\right| \geq\left|\lambda_{2}(A)\right| \geq \ldots \geq\left|\lambda_{n}(A)\right|$. Let $c^{T}$ be a row vector of order $n$ whose entries are nonnegative numbers that sum to $n$. Define $\mathcal{S}(c)$, to be the set of $n \times n$ row-stochastic matrices with column sum vector $c^{T}$. In this paper the quantity $\overline{\lambda(c)}=\max \left\{\mid \lambda_{2}(A) \| A \in \mathcal{S}(c)\right\}$ is considered. The vectors $c^{T}$ such that $\overline{\lambda(c)}<1$ are identified and in those cases, nontrivial upper bounds on $\overline{\lambda(c)}$ and weak ergodicity results for forward products are provided. The results are obtained via a mix of analytic and combinatorial techniques.
\end{abstract}

Key words. Stochastic matrix, Subdominant eigenvalue, Bipartite graph.

AMS subject classifications. 15A51, 15A18, 15A42, 60J10.

1. Introduction. An $n \times n$, entrywise nonnegative matrix $A$ is stochastic if each of its row sums is equal to 1 . Since that row sum condition can be written as $A \mathbf{1}=\mathbf{1}$, where $\mathbf{1}$ denotes an all-ones vector of the appropriate order, we find that 1 is an eigenvalue of $A$. It follows from the Perron-Frobenius theorem (see [9]) that for any eigenvalue $\lambda$ of $A$, we have $|\lambda| \leq 1$, so that in fact 1 is a dominant eigenvalue for $A$. Given a stochastic matrix $A$ of order $n$, we denote its eigenvalues as $1=\lambda_{1}(A), \lambda_{2}(A), \ldots, \lambda_{n}(A)$, ordered so that $1=\left|\lambda_{1}(A)\right| \geq\left|\lambda_{2}(A)\right| \geq \ldots \geq\left|\lambda_{n}(A)\right|$. We refer to $\lambda_{2}(A)$ as a subdominant eigenvalue of $A$.

Associated with any stochastic matrix $A$ is a corresponding Markov chain, i.e. a sequence of row vectors $x_{k}^{T}, k=0,1,2, \ldots$, where $x_{0}^{T}$ is a nonnegative vector with entries summing to 1 , and where the vectors satisfy the recurrence relation $x_{k}^{T}=$ $x_{k-1}^{T} A, k \in \mathbb{N}$. If it happens that $\left|\lambda_{2}(A)\right|<1$, it is straightforward to determine that the sequence $x_{k}^{T}$ converges as $k \rightarrow \infty$, with limit $y^{T}$, where $y^{T}$ is the left eigenvector of $A$ corresponding to the eigenvalue 1 , normalised so that $y^{T} \mathbf{1}=1$. Moreover, in that case, the asymptotic rate of convergence of the sequence $x_{k}^{T}$ is governed by $\left|\lambda_{2}(A)\right|$. We note in passing that $\left|\lambda_{2}(A)\right|<1$ if and only if the matrix $A$ has a single aperiodic essential class of indices (see [9] for the necessary definitions).

\footnotetext{
* Received by the editors August 16, 2009. Accepted for publication December 9, 2009. Handling Editor: Michael Neumann.

${ }^{\dagger}$ Hamilton Institute, National University of Ireland Maynooth, Ireland (stephen.kirkland@nuim.ie). Research supported in part by the SFI Stokes Programme.
} 
On the other hand, if $\left|\lambda_{2}(A)\right|=1$, then the sequence of iterates $x_{k}^{T}$ may fail to converge, or it may converge to a limit that is dependent upon the initial vector $x_{0}^{T}$. Thus we find that the modulus of the subdominant eigenvalue of a stochastic matrix is critical in determining the long-term behaviour of the corresponding Markov chain. Because of that fact, there is a body of work on estimating the modulus of the subdominant eigenvalue for a stochastic matrix; see for instance $[4,5,7,8]$.

Letting $e_{l}$ denote the $l$-th standard unit basis vector in $\mathbb{R}^{n}$, we have the following particularly useful bound which is attributed to Dobrushin [2]; proofs can be found in [1] and [9].

Proposition 1.1. Let $A$ be a stochastic matrix of order $n$. Define $\tau(A)$ as follows:

$$
\tau(A)=\frac{1}{2} \max _{i, j=1, \ldots, n}\left\|e_{i}^{T} A-e_{j}^{T} A\right\|_{1}
$$

Then $\left|\lambda_{2}(A)\right| \leq \tau(A)$.

We note that $\tau(A)$ can be written equivalently as $\tau(A)=\frac{1}{2} \max _{i, j}\left\{\sum_{k=1}^{n} \mid A_{i k}-\right.$ $\left.A_{j k} \mid\right\}$, or as $\tau(A)=1-\min _{i, j} \sum_{k=1}^{n} \min \left\{A_{i k}, A_{j k}\right\}$ ([9]). A stochastic matrix $A$ is called a scrambling matrix if $\tau(A)<1$.

In this paper, we investigate bounds on the subdominant eigenvalue of a stochastic matrix $A$ in terms of its column sum vector $c^{T}=\mathbf{1}^{T} A$. The following example helps to motivate the problem.

Example 1.2. Let $c^{T}=\left[\begin{array}{ccc}\frac{3}{2} & \frac{3}{4} & \frac{3}{4}\end{array}\right]$, and suppose that $A$ is a stochastic matrix with column sum vector $c^{T}$. We claim then that $\left|\lambda_{2}(A)\right|<1$.

To verify the claim, first observe that the sum of the entries $a_{22}+a_{23}+a_{32}+a_{33}$ is bounded above by $\frac{3}{2}$, so it follows that no subset of $\{2,3\}$ can yield an essential class of indices. Hence we see that there is a single essential class, and that this class necessarily contains index 1 .

If the essential class is periodic, then its period is 2 or 3 . Evidently the latter is impossible, otherwise $A$ would be a cyclic permutation matrix, and so would fail to have column sum vector $c^{T}$. If the essential class is periodic with period 2 and contains just two indices, then $\left[\begin{array}{ll}0 & 1 \\ 1 & 0\end{array}\right]$ is a principal submatrix of $A$, again contradicting the column sum condition. Finally, if $A$ is irreducible and periodic with period 2, then it must have the form

$$
\left[\begin{array}{ccc}
0 & a_{12} & a_{13} \\
1 & 0 & 0 \\
1 & 0 & 0
\end{array}\right],
$$


and again the column sum condition is violated. We conclude that any stochastic matrix $A$ with column sum vector $c^{T}$ has a single aperiodic essential class, and so $\left|\lambda_{2}(A)\right|<1$.

The example above prompts our interest in the following line of investigation: what can be said about the modulus of a subdominant eigenvalue of a stochastic matrix based on its vector of column sums?

It is not difficult to see that for a vector $c \in \mathbb{R}^{n}$, the row vector $c^{T}$ serves as the vector of column sums for some $n \times n$ stochastic matrix if and only if the entries in $c^{T}$ are nonnegative and sum to $n$. In the sequel it will be convenient to take the entries in such a vector to be in nonincreasing order, prompting the following definition: we say that a row vector $c^{T}$ with $n$ entries is an admissible column sum vector of order $n$ provided that $c_{1} \geq c_{2} \geq \ldots \geq c_{n} \geq 0$ and $\sum_{i=1}^{n} c_{i}=n$. Given an admissible column sum vector of order $n$, we let $\mathcal{S}(c)$ denote the set of $n \times n$ stochastic matrices $A$ such that $\mathbf{1}^{T} A=c^{T}$. Evidently $\mathcal{S}(c) \neq \emptyset$ since $\frac{1}{n} \mathbf{1} c^{T} \in \mathcal{S}(c)$, and it is straightforward to see that $\mathcal{S}(c)$ is a compact and convex polytope. Finally, given an admissible column sum vector $c^{T}$ of order $n$, we define $\overline{\lambda_{2}(c)}$ as

$$
\overline{\lambda_{2}(c)}=\max \left\{\left|\lambda_{2}(A)\right| \mid A \in \mathcal{S}(c)\right\} .
$$

In this paper, we consider admissible column sum vectors $c^{T}$ of order $n \geq 3$ (the case $n=2$ is not especially interesting), and identify all such $c^{T}$ for which $\overline{\lambda_{2}(c)}<1$. It is perhaps not too surprising that there are several classes of admissible column sum vectors $c^{T}$ for which $\overline{\lambda_{2}(c)}=1$. However, there are three classes of admissible column sum vectors $c^{T}$ for which $\overline{\lambda_{2}(c)}<1$, and for each of those classes we provide a nontrivial (and in one case, attainable) upper bound for $\overline{\lambda_{2}(c)}$.

We will employ the following notation. For a matrix $A$, its entries will be denoted by $A_{i j}$, while the entries in the $m$-th power of $A$ will be denoted $\left(A^{m}\right)_{i j}$; similarly, entries in a matrix product $A B$ are denoted $(A B)_{i j}$. We use $O$ to denote a zero matrix or vector, and the order will be clear from the context.

Throughout, we rely on standard results for stochastic matrices, as well as some basic ideas from graph theory. The reader is referred to [9] for background on the former and to [6] for results on the latter.

2. Preliminary results. We begin by considering the function $\tau$.

Proposition 2.1. Suppose that $c^{T}$ is an admissible column sum vector of order $n \geq 3$.

a) If $c_{1} \geq n-1$, then $\max \{\tau(A) \mid A \in \mathcal{S}(c)\}=n-c_{1}$.

b) If $c_{1}<n-1$, then $\max \{\tau(A) \mid A \in \mathcal{S}(c)\}=1$. 
Proof. a) Suppose that $A \in \mathcal{S}(c)$. Fix an index $i$ between 1 and $n$. Then $A_{i 1}=1-\sum_{j=2}^{n} A_{i j} \geq 1-\sum_{j=2}^{n} c_{j}=1-\left(n-c_{1}\right)=c_{1}-(n-1)$. Thus we have $A e_{1} \geq\left(c_{1}-(n-1)\right) \mathbf{1}$, and we find readily that $\tau(A) \leq n-c_{1}$.

It remains only to show that the upper bound on $\tau$ is attained. To see this, consider the matrix $A$ given by

$$
A=\left[\begin{array}{c|ccc}
1-\sum_{j=2}^{n} c_{j} & c_{2} & \ldots & c_{n} \\
\hline \mathbf{1} & & O
\end{array}\right] ;
$$

it is straightforward to determine that $A \in \mathcal{S}(c)$ and that $\tau(A)=n-c_{1}$.

b) Since $c_{1}<n-1$, we have $\sum_{j=2}^{n} c_{j}>1$. Set $\sigma=\sum_{j=2}^{n} c_{j}$, and let $\tilde{c}^{T}=$ $\left[\begin{array}{lll}c_{2} & \ldots & c_{n}\end{array}\right]$. Consider the matrix

$$
B=\left[\begin{array}{c|c}
1 & O^{T} \\
0 & \frac{1}{\sigma} \tilde{c}^{T} \\
\hline\left(\frac{c_{1}-1}{n-2}\right) \mathbf{1} & \left(\frac{\sigma-1}{(n-2) \sigma}\right) \mathbf{1} \tilde{c}^{T}
\end{array}\right] .
$$

It is readily seen that $B \in \mathcal{S}(c)$ and that $\tau(B)=1$.

The following result provides the value of $\overline{\lambda_{2}(c)}$ for a particular class of admissible column sum vectors.

COROLlaRY 2.2. Let $c^{T}$ be an admissible column sum vector of order $n \geq 3$, and suppose that $c_{1} \geq n-1$. Then $\overline{\lambda_{2}(c)}=n-c_{1}$.

Proof. From Proposition 2.1, we see that for any $A \in \mathcal{S}(c)$ we have $\left|\lambda_{2}(A)\right| \leq$ $\tau(A)=n-c_{1}$. Next, we consider the following matrix:

$$
A=\left[\begin{array}{c|ccc}
1-\sum_{j=2}^{n} c_{j} & c_{2} & \ldots & c_{n} \\
\hline \mathbf{1} & & O
\end{array}\right] .
$$

It is readily seen that the eigenvalues of $A$ are $1,-\sum_{j=2}^{n} c_{j}$, and 0 with multiplicity $n-2$. Hence $\lambda_{2}(A)=-\sum_{j=2}^{n} c_{j}$ so that $\left|\lambda_{2}(A)\right|=\sum_{j=2}^{n} c_{j}=n-c_{1}$. The conclusion now follows.

Given a sequence of $n \times n$ stochastic matrices $A_{1}, A_{2}, \ldots$, we have the corresponding sequence of forward products, $T_{k}=A_{1} \ldots A_{k}, k \in \mathbb{N}$. These forward products arise naturally in the nonhomogeneous Markov chain associated with the sequence $A_{j}, j \in \mathbb{N}$. The sequence of $n \times n$ forward products $T_{k}, k \in \mathbb{N}$ is weakly ergodic if, for each $i, j, l=1, \ldots, n$, we have $\left(T_{k}\right)_{i l}-\left(T_{k}\right)_{j l} \rightarrow 0$ as $k \rightarrow \infty$. As is shown in [9], weak ergodicity of the sequence of forward products $T_{k}$ is equivalent to the condition that $\tau\left(T_{k}\right) \rightarrow 0$ as $k \rightarrow \infty$.

Our next remark deals with weak ergodicity for a certain class of forward products. 
Remark 2.3. According to Proposition 2.1, if $c_{1}>n-1$, then for each $A \in$ $\mathcal{S}(c), \tau(A) \leq n-c_{1}<1$. Consequently, for any sequence of matrices $A_{1}, A_{2}, \ldots \in$ $\mathcal{S}(c)$, the sequence of forward products $T_{k}=A_{1} \ldots A_{k}, k \in \mathbb{N}$ has the property that $\tau\left(T_{k}\right) \leq\left(n-c_{1}\right)^{k}$, so that $\tau\left(T_{k}\right) \rightarrow 0$ as $k \rightarrow \infty$. Thus, for any sequence $A_{j} \in \mathcal{S}(c)$, the corresponding sequence of forward products is weakly ergodic.

Our next few results identify classes of admissible column sum vectors $c^{T}$ for which $\overline{\lambda_{2}(c)}=1$.

Proposition 2.4. Suppose that $c^{T}$ is an admissible column sum vector of order $n \geq 3$, and that $c_{2} \geq 1$. Then $\overline{\lambda_{2}(c)}=1$.

Proof. It suffices to construct a matrix $A \in \mathcal{S}(c)$ having a non-Perron eigenvalue of modulus 1 . If $c^{T}=\mathbf{1}^{T}$, we can take $A$ to be the $n \times n$ cyclic permutation matrix with $A_{i i+1}=1, i=1, \ldots, n-1$ and $A_{n 1}=1$; in that case, the eigenvalues are just the $n$-th roots of unity, yielding the desired conclusion.

If $c^{T} \neq \mathbf{1}^{T}$, then necessarily $c_{n}<1$. Select the index $j$ such that $c_{j} \geq 1>c_{j+1}$, and observe that $j$ is between 2 and $n-1$. Set $u^{T}=\left[\begin{array}{lll}c_{1}-1 & \ldots & c_{j}-1\end{array}\right], v^{T}=$ $\left[\begin{array}{ccc}c_{j+1} & \ldots & c_{n}\end{array}\right]$, and let $P$ be a $j \times j$ cyclic permutation matrix. Consider the matrix $A$ given by

$$
A=\left[\begin{array}{c|c}
P & O \\
\hline \frac{1}{n-j} \mathbf{1} u^{T} & \frac{1}{n-j} \mathbf{1} v^{T}
\end{array}\right] .
$$

Evidently $A \in \mathcal{S}(c)$ and has the $j$-th roots of unity among its eigenvalues. We conclude that $\overline{\lambda_{2}(c)}=1$.

Lemma 2.5. Let $c^{T}$ be an admissible column sum vector of order $n \geq 3$. Suppose that there is an $m \in \mathbb{N}$ with $m \leq n-2$ such that $c_{1} \geq m$ and $\sum_{j=2}^{m+1} c_{j} \geq 1$. Then $\overline{\lambda_{2}(c)}=1$.

Proof. In view of Proposition 2.4, it suffices to consider the case that $c_{2}<1$. Note that necessarily $n \geq 4$ in that case. From our hypothesis, there is an index $j$ such that $c_{2}+\ldots+c_{j}<1 \leq c_{2}+\ldots+c_{j+1}$, and note that necessarily $j \leq m$.

Next, we construct a matrix in $\mathcal{S}(c)$ for which the subdominant eigenvalue has modulus 1 . To do so, let $T$ be the matrix of order $j+1$ given by

$$
T=\left[\begin{array}{c|cccc}
0 & c_{2} & \ldots & c_{j} & \left(1-\sum_{l=2}^{j} c_{l}\right) \\
\hline \mathbf{1} & \multicolumn{4}{c}{O}
\end{array}\right]
$$

We take $u^{T}$ to be the vector $u^{T}=\left[\begin{array}{llllll}\left(c_{1}-j\right) & 0 & \ldots & 0 & \left(\sum_{l=2}^{j+1} c_{l}-1\right)\end{array}\right]$, and $v^{T}$ 
to be the vector $v^{T}=\left[\begin{array}{lll}c_{j+2} & \ldots & c_{n}\end{array}\right]$. Finally, we let $A$ be given by

$$
A=\left[\begin{array}{c|c}
T & O \\
\hline \frac{1}{n-j-1} \mathbf{1} u^{T} & \frac{1}{n-j-1} \mathbf{1} v^{T}
\end{array}\right] .
$$

Since $T$, and hence $A$, has -1 as an eigenvalue, we see that $\overline{\lambda_{2}(c)}=1$, as desired.

Proposition 2.6. Suppose that $c^{T}$ is an admissible column sum vector of order $n \geq 5$, and that $n-2>c_{1} \geq 2$. Then $\overline{\lambda_{2}(c)}=1$.

Proof. Let $m=\left\lfloor c_{1}\right\rfloor$, so that $n-3 \geq m \geq 2$. We have $m+3 \leq n$, and since the $c_{i}$ 's are nonincreasing and sum to $n$, we have $c_{1}+c_{2}+\ldots+c_{m+1}+c_{m+2}+c_{m+3} \geq m+3$. Since $c_{1}<m+1$, it follows that $c_{2}+\ldots+c_{m+1}+c_{m+2}+c_{m+3}>2$. Again, since the $c_{i}$ 's are nonincreasing and $m \geq 2$, we find that $c_{2}+\ldots+c_{m+1} \geq c_{m+2}+c_{m+3}$; it now follows that $c_{2}+\ldots+c_{m+1}>1$. Thus, $c_{1} \geq m$ and $\sum_{j=2}^{m+1} c_{j} \geq 1$, so the hypotheses of Lemma 2.5 are satisfied; the conclusion now follows.

In view of Corollary 2.2, Proposition 2.4, Lemma 2.5 and Proposition 2.6, it remains only to consider admissible column sum vectors $c^{T}$ of order $n$ satisfying one of the following two sets of conditions:

Case i) $n-1>c_{1}$ and $\sum_{j=2}^{n-1} c_{j}<1$; and

Case ii) $2>c_{1}>1$ and $c_{j}<1, j=2, \ldots, n$.

We deal with Cases i) and ii) in Sections 3 and 4, respectively.

3. A bound for Case i). Throughout this section, unless otherwise indicated, we take $c^{T}$ to be an admissible column sum vector of order $n \geq 4$ such that $n-1>c_{1}$ and $\sum_{j=2}^{n-1} c_{j}<1$. Observe that since $\sum_{j=2}^{n} c_{j}=n-c_{1}$, we have $c_{n} \leq \frac{n-c_{1}}{n-1}$, so that $1>\sum_{j=2}^{n-1} c_{j} \geq \frac{(n-2)\left(n-c_{1}\right)}{n-1}$. It now follows that $c_{1}>n-1-\frac{1}{n-2}$, so that in fact $c_{1}>n-\frac{3}{2}$.

We proceed by providing bounds on $\tau\left(A^{2}\right)$ when $A \in \mathcal{S}(c)$ in Propositions 3.1 and 3.3 below.

Proposition 3.1. Suppose that $A \in \mathcal{S}(c)$, that $n \geq 4$, and that $A_{11}=0$. Then $\tau\left(A^{2}\right) \leq 1-\left(n-1-c_{1}\right)\left(1-\sum_{j=2}^{n-1} c_{j}\right)$.

Proof. Let $\tilde{c}^{T}=\left[\begin{array}{lll}c_{2} & \ldots & c_{n}\end{array}\right]$, and let $B$ denote the principal submatrix of $A$ on rows and columns $2, \ldots, n$. Then $A$ can be written as

$$
A=\left[\begin{array}{c|c}
0 & \tilde{c}^{T}-\mathbf{1}^{T} B \\
\hline \mathbf{1}-B \mathbf{1} & B
\end{array}\right] .
$$

We have $c_{1}=n-1-\mathbf{1}^{T} B \mathbf{1}$, so that $\mathbf{1}^{T} B \mathbf{1}=n-1-c_{1}>0$. Since $c_{1}>n-2$, we see that for each $l=2, \ldots, n, A_{l 1}=c_{1}-\sum_{2 \leq j \leq n, j \neq l} A_{j 1} \geq c_{1}-(n-2)$. Also, for each $l=2, \ldots, n$ we have $A_{1 l}=1-\sum_{2 \leq j \leq n, j \neq l} A_{1 j} \geq 1-\sum_{2 \leq j \leq n, j \neq l} c_{j} \geq 1-\sum_{j=2}^{n-1} c_{j}$. 
Fix an index $l$ between 2 and $n$. For any index $i$ between 2 and $n$, we have $\left(A^{2}\right)_{i l} \geq A_{i 1} A_{1 l} \geq\left(c_{1}-(n-2)\right)\left(1-\sum_{j=2}^{n-1} c_{j}\right)$. Also, $\left(A^{2}\right)_{1 l}=\sum_{j=2}^{n} A_{1 j} A_{j l} \geq$ $\left(1-\sum_{j=2}^{n-1} c_{j}\right) \sum_{j=2}^{n} A_{j l}=\left(1-\sum_{j=2}^{n-1} c_{j}\right) \mathbf{1}^{T} B e_{l}$. Since $n \geq 4$ we have $c_{1}>n-\frac{3}{2}$, which yields the inequality $n-1-c_{1}<c_{1}-(n-2)$. Hence we find that $\mathbf{1}^{T} B e_{l} \leq$ $\mathbf{1}^{T} B \mathbf{1}=n-1-c_{1}<c_{1}-(n-2)$.

Thus we find that for each $i=1, \ldots, n$ and $l=2, \ldots, n$,

$$
\left(A^{2}\right)_{i l} \geq\left(1-\sum_{j=2}^{n-1} c_{j}\right) \mathbf{1}^{T} B e_{l}
$$

so that $A^{2} e_{l} \geq\left(1-\sum_{j=2}^{n-1} c_{j}\right)\left(\mathbf{1}^{T} B e_{l}\right) \mathbf{1}$. It now follows that $\tau\left(A^{2}\right) \leq 1-(1-$ $\left.\sum_{j=2}^{n-1} c_{j}\right) \sum_{l=2}^{n} \mathbf{1}^{T} B e_{l}=1-\left(1-\sum_{j=2}^{n-1} c_{j}\right) \mathbf{1}^{T} B \mathbf{1}=1-\left(1-\sum_{j=2}^{n-1} c_{j}\right)\left(n-1-c_{1}\right)$.

ExAMPLE 3.2. It turns out that equality can hold in the bound of Proposition 3.1. For the matrix

$$
A=\left[\begin{array}{c|ccc|c}
0 & c_{2} & \ldots & c_{n-1} & 1-\sum_{j=2}^{n-1} c_{j} \\
\hline \mathbf{1} & \multicolumn{3}{|c|}{O} & O \\
\hline c_{1}-n+2 & \multicolumn{3}{|c|}{O^{T}} & n-1-c_{1}
\end{array}\right],
$$

a straightforward computation shows that $\tau\left(A^{2}\right)=1-\left(n-1-c_{1}\right)\left(1-\sum_{j=2}^{n-1} c_{j}\right)$.

Proposition 3.3. Suppose that $A \in \mathcal{S}(c)$, that $n \geq 4$, and that $A_{k 1}=0$ for some $k \neq 1$. Then $\tau\left(A^{2}\right) \leq 1-\left(n-1-c_{1}\right)\left(1-\sum_{j=2}^{n-1} c_{j}\right)$.

Proof. As in the proof of Proposition 3.1, we have $A_{j 1} \geq c_{1}-(n-2)$ for each $j \neq k$. Hence, for all such $j$ we find that $\left(A^{2}\right)_{j 1} \geq A_{j 1} A_{11} \geq\left(c_{1}-(n-2)\right)^{2}$. Also, $\left(A^{2}\right)_{k 1}=\sum_{1 \leq j \leq n, j \neq k} A_{k j} A_{j 1} \geq\left(c_{1}-(n-2)\right) \sum_{1 \leq j \leq n, j \neq k} A_{k j}=\left(c_{1}-(n-2)\right)(1-$ $\left.A_{k k}\right) \geq\left(c_{1}-(n-2)\right)\left(1-c_{k}\right) \geq\left(c_{1}-(n-2)\right)\left(1-\sum_{j=2}^{n-1} c_{j}\right)$. Note that since $n \geq$ $4, c_{1}-(n-2) \geq n-1-c_{1}$, and since $\sum_{j=1}^{n-1} c_{j} \geq n-1$, we have $c_{1}-(n-2) \geq 1-\sum_{j=2}^{n-1} c_{j}$. It now follows that $A^{2} e_{1} \geq\left(1-\sum_{j=2}^{n-1} c_{j}\right)\left(n-1-c_{1}\right) \mathbf{1}$, which yields the conclusion. $\mathrm{C}$

Here is one of the main results of this section.

Theorem 3.4. Suppose that $n \geq 4$, and that $A \in \mathcal{S}(c)$. Then

$$
\tau\left(A^{2}\right) \leq 1-\left(n-1-c_{1}\right)\left(1-\sum_{j=2}^{n-1} c_{j}\right) .
$$

Proof. If $A$ has a zero in the first column, then the inequality follows from Propositions 3.1 and 3.3. Suppose now that $A e_{1}>0$, and let $t$ denote the smallest entry in $A e_{1}$. Write $A$ as $A=t \mathbf{1} e_{1}^{T}+(1-t) B$, and note that $B$ is a stochastic matrix 
with a zero in its first column. Note also that $\tau\left(A^{2}\right)=(1-t)^{2} \tau\left(B^{2}\right)$. The vector of column sums for $B$ is $\left[\begin{array}{llll}\frac{c_{1}-n t}{1-t} & \frac{c_{2}}{1-t} & \ldots & \frac{c_{n}}{1-t}\end{array}\right]$.

Since $c_{1}<n-1$, we find that that $n-1>\frac{c_{1}-n t}{1-t}$. Suppose first that $\frac{\sum_{j=2}^{n-1} c_{j}}{1-t}<1$. Then $B$ satisfies the hypotheses of Propositions 3.1 and 3.3, so we find that $\tau\left(A^{2}\right)=$ $(1-t)^{2} \tau\left(B^{2}\right) \leq(1-t)^{2}-\left(1-\sum_{j=2}^{n-1} c_{j}-t\right)\left(n-1-c_{1}+t\right)=1-\left(n-1-c_{1}\right)(1-$ $\left.\sum_{j=2}^{n-1} c_{j}\right)+t\left(n-c_{1}-4+\sum_{j=2}^{n-1} c_{j}+2 t\right)$. From an argument similar to the one given at the beginning of this section, we find that $\frac{c_{1}-n t}{1-t} \geq n-2$. Hence $2 t \leq c_{1}-n+2$, so that $n-c_{1}-4+\sum_{j=2}^{n-1} c_{j}+2 t \leq-2+\sum_{j=2}^{n-1} c_{j}<0$. The desired inequality on $\tau\left(A^{2}\right)$ now follows.

Next, suppose that $\sum_{j=2}^{n-1} c_{j} \geq 1-t$. We then have $\tau\left(A^{2}\right) \leq(1-t)^{2} \leq\left(\sum_{j=2}^{n-1} c_{j}\right)^{2}$, and we claim that $\left(\sum_{j=2}^{n-1} c_{j}\right)^{2} \leq 1-\left(n-1-c_{1}\right)\left(1-\sum_{j=2}^{n-1} c_{j}\right)$. The claim is equivalent to the inequality $\left(n-1-c_{1}\right)\left(1-\sum_{j=2}^{n-1} c_{j}\right) \leq 1-\left(\sum_{j=2}^{n-1} c_{j}\right)^{2}$, which in turn simplifies to $n-1-c_{1} \leq 1+\sum_{j=2}^{n-1} c_{j}$. As $\sum_{j=1}^{n-1} c_{j} \geq n-1$, the claim follows immediately.

口

The following is immediate from Theorem 3.4.

COROLlary 3.5. If $c^{T}$ is an admissible column sum vector of order $n \geq 4$ with $n-1>c_{1}$ and $\sum_{j=2}^{n-1} c_{j}<1$, then $\overline{\lambda_{2}(c)} \leq \sqrt{1-\left(n-1-c_{1}\right)\left(1-\sum_{j=2}^{n-1} c_{j}\right)}$.

REMARK 3.6. We note that in the results above in this section, the hypothesis that $n \geq 4$ was used only to establish that $n-1-c_{1} \leq c_{1}-(n-2)$. A minor modification of the arguments above yields the following:

If $c^{T}$ is an admissible column sum vector of order 3 with $2>c_{1}>1$ and $c_{2}<1$, then for any $A \in \mathcal{S}(c)$, we have $\tau\left(A^{2}\right) \leq 1-\left(1-c_{2}\right) \min \left\{2-c_{1}, c_{1}-1\right\}$. Further, $\overline{\lambda_{2}(c)} \leq \sqrt{1-\left(1-c_{2}\right) \min \left\{2-c_{1}, c_{1}-1\right\}}$.

The next example yields a lower bound on $\overline{\lambda_{2}(c)}$.

EXAMPLE 3.7. Let $c^{T}$ be an admissible column sum vector of order $n \geq 3$ such that $n-1>c_{1}$ and $\sum_{j=2}^{n-1} c_{j}<1$. Consider the matrix $A$ given by

$$
A=\left[\begin{array}{c|ccc|c}
0 & c_{2} & \ldots & c_{n-1} & 1-\sum_{j=2}^{n-1} c_{j} \\
\hline \mathbf{1} & \multicolumn{3}{|c|}{O} & O \\
\hline c_{1}-n+2 & \multicolumn{3}{|c|}{O^{T}} & n-1-c_{1}
\end{array}\right] .
$$

Evidently $A \in \mathcal{S}(c)$, and a straightforward computation shows that the eigenvalues of $A$ are: 1,0 (with multiplicity $n-3$ ) and

$$
\frac{1}{2}\left(-\left(c_{1}-(n-2)\right) \pm \sqrt{\left(c_{1}-(n-2)\right)^{2}+4 \sum_{j=2}^{n-1} c_{j}\left(n-1-c_{1}\right)}\right) .
$$


In particular we see that

$$
\left|\lambda_{2}(A)\right|=\frac{1}{2}\left(\left(c_{1}-(n-2)\right)+\sqrt{\left(c_{1}-(n-2)\right)^{2}+4 \sum_{j=2}^{n-1} c_{j}\left(n-1-c_{1}\right)}\right) .
$$

Hence we have $\left|\lambda_{2}(A)\right|>c_{1}-(n-2)>1-\frac{1}{n-2}$, so that $\overline{\lambda_{2}(c)}>1-\frac{1}{n-2}$.

Next, we provide a weak ergodicity result for forward products of matrices in $\mathcal{S}(c)$. In order to do so, we need to discuss the extreme points of the convex polytope $\mathcal{S}(c)$, that is, those matrices in $\mathcal{S}(c)$ that cannot be expressed as a nontrivial convex combination of other matrices in $\mathcal{S}(c)$. Recall that for an $n \times n$ stochastic matrix $A$, the bipartite graph $\mathcal{B}$ associated with $A$ is the graph on $2 n$ vertices with the following structure: there are $n$ row vertices labeled $\mathcal{R} 1, \ldots, \mathcal{R} n$, and $n$ column vertices labeled $\mathcal{C} 1, \ldots, \mathcal{C} n$, and $\mathcal{B}$ contains an edge between $\mathcal{R} i$ and $\mathcal{C} j$ if and only if $A_{i j}>0$. We use the notation $\mathcal{R} i \sim \mathcal{C} j$ to denote such an edge.

Our discussion of extreme points of $\mathcal{S}(c)$ makes use of this bipartite graph. In particular, it is known that a matrix $A \in \mathcal{S}(c)$ is an extreme point if and only if its associated bipartite graph contains no cycles (this follows from a more general result for so-called transportation polytopes; see [10]).

TheOREm 3.8. Suppose that $c^{T}$ is an admissible column sum vector of order $n \geq 4$ with $n-1>c_{1}$ and $\sum_{j=2}^{n-1} c_{j}<1$. Let $A_{k}, k \in \mathbb{N}$ be a sequence of matrices in $\mathcal{S}(c)$. Then the sequence of forward products $T_{k}=A_{1} A_{2} \ldots A_{k}, k \in \mathbb{N}$, is weakly ergodic.

Proof. It is sufficient to show that there is a scalar $0 \leq r<1$ such that for any pair of matrices $A, B \in \mathcal{S}(c), \tau(A B) \leq r$. We proceed by establishing the existence of such an $r$.

First, observe that it is enough to prove that the inequality holds for any pair of matrices that are extreme points of $\mathcal{S}(c)$. This follows from the fact that any pair of matrices $A, B \in \mathcal{S}(c)$ can be written as a convex combination of matrices of the form $\tilde{A} \tilde{B}$ where $\tilde{A}$ and $\tilde{B}$ are extreme points of $\mathcal{S}(c)$. As noted above, a matrix in $\mathcal{S}(c)$ is an extreme point if and only if its associated bipartite graph contains no cycles.

Henceforth, we take $A$ and $B$ to be extreme points of $\mathcal{S}(c)$. Suppose that $A$ has a positive first column. Then since the bipartite graph for $A$ has no cycles, we find that for each $j=2, \ldots, n$, the $j$-th column of $A$ contains a single positive entry, $c_{j}$. It now follows that the first column contains a 1 in some position, and that in the remaining positions, the entries in the first column of $A$ are of the form $1-c_{j}$ for some $j \geq 2$. It follows readily that $\tau(A) \leq c_{2}$. Thus we have $\tau(A B) \leq \tau(A) \leq c_{2}$. A similar argument holds if $B$ has a positive first column. 
Next we suppose that each of $A$ and $B$ has a zero in the first column, say with $A_{i_{0} 1}=0, B_{j_{0} 1}=0$. Then for each $i \neq i_{0}$ and $j \neq j_{0}$, we have $A_{i 1}, B_{j 1} \geq c_{1}-(n-2)$. Also, for any index $l \geq 2, A_{i_{0} l}=1-\sum_{2 \leq p \leq n, p \neq l} A_{i_{0} p} \geq 1-\sum_{2 \leq p \leq n, p \neq l} c_{p} \geq 1-$ $\sum_{p=2}^{n-1} c_{p}$. Similarly, $B_{j_{0} l} \geq 1-\sum_{p=2}^{n-1} c_{p}$. Thus we find that $A \geq\left(c_{1}-(n-2)\right)(\mathbf{1}-$ $\left.e_{i_{0}}\right) e_{1}^{T}+\left(1-\sum_{p=2}^{n-1} c_{p}\right) e_{i_{0}}\left(\mathbf{1}^{T}-e_{1}^{T}\right)$ and that $B e_{1} \geq\left(c_{1}-(n-2)\right)\left(\mathbf{1}-e_{j_{0}}\right)$. In the case that $j_{0} \neq 1$, we then find that $A B e_{1} \geq\left(c_{1}-(n-2)\right)^{2}\left(\mathbf{1}-e_{i_{0}}\right)+\left(1-\sum_{p=2}^{n-1} c_{p}\right)\left(c_{1}-\right.$ $(n-2))(n-2) e_{i_{0}}$, from which we find that $\tau(A B) \leq 1-\left(c_{1}-(n-2)\right) \min \left\{c_{1}-(n-\right.$ 2), $\left.(n-2)\left(1-\sum_{p=2}^{n-1} c_{p}\right)\right\}$.

On the other hand, if $j_{0}=1$, then we may write $B$ as

$$
B=\left[\begin{array}{c|c}
0 & \tilde{c}^{T}-\mathbf{1}^{T} \tilde{B} \\
\hline \mathbf{1}-\tilde{B} \mathbf{1} & \tilde{B}
\end{array}\right],
$$

where $\tilde{c}^{T}$ is the subvector of $c^{T}$ on columns $2, \ldots, n$. Note that $\mathbf{1}^{T} \tilde{B} \mathbf{1}=n-1-c_{1}>0$. Fix an index $l$ between 2 and $n$. If $i \neq i_{0}$, we have $(A B)_{i l} \geq A_{i 1} B_{1 l} \geq\left(c_{1}-(n-\right.$ $2))\left(1-\sum_{p=2}^{n-1} c_{p}\right)$. Also, $(A B)_{i_{0} l}=\sum_{j=2}^{n} A_{i_{0} j} B_{j l} \geq\left(1-\sum_{p=2}^{n-1} c_{p}\right) \sum_{j=2}^{n} B_{j l}=(1-$ $\left.\sum_{p=2}^{n-1} c_{p}\right) \mathbf{1}^{T} \tilde{B} e_{l}$. As in Proposition 3.1, we have $c_{1}-(n-2) \geq \mathbf{1}^{T} \tilde{B} e_{l}$, so we find that $A B e_{l} \geq\left(1-\sum_{p=2}^{n-1} c_{p}\right)\left(\mathbf{1}^{T} \tilde{B} e_{l}\right) \mathbf{1}$. Thus, $\tau(A B) \leq 1-\sum_{l=2}^{n}\left(1-\sum_{p=2}^{n-1} c_{p}\right)\left(\mathbf{1}^{T} \tilde{B} e_{l}\right)=$ $1-\left(1-\sum_{p=2}^{n-1} c_{p}\right)\left(\mathbf{1}^{T} \tilde{B} \mathbf{1}\right)=1-\left(1-\sum_{p=2}^{n-1} c_{p}\right)\left(n-1-c_{1}\right)$.

From the considerations above, it now follows that for any pair of extreme matrices $A, B$ in $\mathcal{S}(c), \tau(A B)$ is bounded above by

$\max \left\{c_{2}, 1-\left(1-\sum_{p=2}^{n-1} c_{p}\right)\left(n-1-c_{1}\right), 1-\left(c_{1}-(n-2)\right)^{2}, 1-\left(c_{1}-(n-2)\right)(n-2)\left(1-\sum_{p=2}^{n-1} c_{p}\right)\right\}$

The conclusion now follows.

REMARK 3.9. The hypothesis that $n \geq 4$ is not essential in the proof of Theorem 3.8. If $c^{T}$ is an admissible column sum vector of order 3 with $2>c_{1}>1$ and $c_{2}<1$, then a minor modification of the proof of that theorem shows that for any sequence of matrices in $\mathcal{S}(c)$, say $A_{k}$, the sequence of forward products $T_{k}=A_{1} A_{2} \ldots A_{k}, k \in \mathbb{N}$, is weakly ergodic.

4. A bound for Case ii). Throughout this section, we consider an admissible column sum vector $c^{T}$ of order $n \geq 3$ having the properties that $2>c_{1}>1$, and $c_{j}<1, j=2, \ldots, n$. Our analysis of this case proceeds by first considering the extreme points of the convex polytope $\mathcal{S}(c)$.

Lemma 4.1. Suppose that $A$ is an extreme point of $\mathcal{S}(c)$. Then its bipartite graph $\mathcal{B}$ is a tree with the properties that a) there is a single row vertex of degree 1 , and $b$ ) all remaining row vertices have degree 2 . Further $\mathcal{B}$ has a perfect matching. 
Proof. If $A$ is an extreme point of $\mathcal{S}(c)$, then its bipartite graph $\mathcal{B}$ must be a forest. Since $c_{1}<2$ and $c_{j}<1, j=2, \ldots, n$, we find that none of columns $2, \ldots, n$ of $A$ can contain a 1 , and that column 1 of $A$ contains at most one 1 . Hence $A$ has at most one row with a single positive entry, so that the number of positive entries in $A$ is at least $2 n-1$. Thus $\mathcal{B}$ has at least $2 n-1$ edges, and since it is necessarily a forest, we deduce that in fact $\mathcal{B}$ has exactly $2 n-1$ edges. Consequently, $\mathcal{B}$ is a tree. Further, we find that in fact precisely one row of $A$ contains a single positive entry (necessarily a 1 , in column 1 ) and all remaining rows of $A$ contain exactly two positive entries. Properties a) and b) for $\mathcal{B}$ now follow.

Next, suppose that we have a tree $T$ on $2 n$ vertices such that its vertex set is partitioned into two subsets, each of cardinality $n$, such that vertices in $T$ are adjacent only if they are in different members of the partition. We now prove that any such tree that also satisfies a) and b) must contain a perfect matching (here we associate the row vertices and column vertices in a) and b) with the partite sets in the bipartition of the vertex set for $T$ ). We proceed by induction on $n$, and note that if $n=1$, then $T=K_{2}$. Suppose now that the result holds for some $m \in \mathbb{N}$, and that $T$ is a tree on $2 m+2$ vertices satisfying a) and b) and whose vertex set bipartition consists of two subsets of cardinality $m+1$. Then $T$ has a pendant row vertex, say $\mathcal{R} i$, and a pendant column vertex, say $\mathcal{C} j$. Observe that $\mathcal{R} i$ and $\mathcal{C} j$ are not adjacent in $T$. Construct a tree $\tilde{T}$ from $T$ by deleting vertices $\mathcal{R} i$ and $\mathcal{C} j$, as well as their incident (pendant) edges. Then $\tilde{T}$ satisfies the hypotheses of the induction, and so contains a perfect matching, say $\tilde{M}$. But then $\tilde{M}$, along with the pendant edges incident with $\mathcal{R} i$ and $\mathcal{C} j$, forms a perfect matching for $T$, completing the induction step.

Next, we find a lower bound on the positive entries in an extreme point of $\mathcal{S}(c)$.

Lemma 4.2. Suppose that $A$ is an extreme point of $\mathcal{S}(c)$. Then the minimum positive entry in $A$ is bounded below by $\min \left\{2-c_{1}, 1-c_{2}\right\}$.

Proof. Let $\mathcal{B}$ denote the bipartite graph of $A$, say with perfect matching $M$, vertex $\mathcal{R} i$ pendant and adjacent to vertex $\mathcal{C} 1$, and vertex $\mathcal{C} j$ pendant and adjacent to vertex $\mathcal{R} k$; note that $j \neq 1$. Observe that the positive entries in the matrix $A$ furnish a weighting of the edges of $\mathcal{B}$ with the properties that: i) the sum of the weights of the edges incident with any row vertex is 1 ; and ii) for each $l=1, \ldots, n$, the sum of the weights of the edges incident with $\mathcal{C l}$ is $c_{l}$.

Suppose that we are given real numbers $z_{1}, \ldots, z_{n}$, and we have a weighting of the edges of $\mathcal{B}$ such that i) the sum of the weights of the edges incident with any row vertex is 1 ; and ii) for each $l=1, \ldots, n$, the sum of the weights of the edges incident with $\mathcal{C l}$ is $z_{l}$. Necessarily we must have $\sum_{l=1}^{n} z_{l}=n$. We claim then that the weights of the edges in the matching $M$ are of the form $\sum_{l=1}^{p_{1}} z_{i_{l}}-p_{2}$, (for some integers $p_{1}, p_{2}$ and some collection of distinct indices $\left.i_{1}, \ldots, i_{p_{1}}\right)$ and that the weights 
of the edges not in $M$ are of the form $q_{2}-\sum_{l=1}^{q_{1}} z_{i_{l}}$ (again for some integers $q_{1}, q_{2}$ and distinct indices $i_{1}, \ldots, i_{q_{1}}$ ). We prove the claim by induction on $n$, and note that the cases $n=1,2$ are straightforward. Suppose now that the claim holds for some $n-1 \in \mathbb{N}$ and that $A$ is of order $n$. Note that the pendant edge incident with vertex $\mathcal{C} j$ has weight $z_{j}$, so that for vertex $\mathcal{R} k$, the edge incident with vertex $\mathcal{R} k$ not in $M$ has weight $1-z_{j}$. For concreteness, we let the column vertex adjacent to $\mathcal{R} k$ where the corresponding edge is not in $M$ be vertex $\mathcal{C} l_{0}$. Now we delete vertex $\mathcal{C} j$, vertex $\mathcal{R} k$, and their incident edges to form $\tilde{\mathcal{B}}$. Observe that the remaining weights yield a weighting of the edges of $\tilde{\mathcal{B}}$ such that the sum of the weights at each row vertex of $\tilde{\mathcal{B}}$ is 1 , and for each $l \neq j$, the sum of the weights at vertex $\mathcal{C} l$ is $\tilde{z}_{l}$, where $\tilde{z}_{l}=z_{l}$ if $l \neq l_{0}$, and $\tilde{z}_{l_{0}}=z_{l_{0}}-\left(1-z_{j}\right)=z_{l_{0}}+z_{j}-1$. Applying the induction hypothesis to $\tilde{\mathcal{B}}$, we find that the edge weights for $\tilde{\mathcal{B}}$ are of the desired form, and hence so are the edge weights for $\mathcal{B}$. This completes the proof of the claim.

Next, suppose that we have an entry in $A$ that is in the open interval $(0,1)$. Let $x_{1}, \ldots, x_{n}$ denote the sequence $c_{1}-1, c_{2}, \ldots, c_{n}$, arranged in nonincreasing order. Then $\sum_{i=1}^{n} x_{i}=n-1$, and each $x_{i} \in(0,1)$. From our claim above, each entry of $A$ in $(0,1)$ is of the form $\sum_{l=1}^{p_{1}} x_{i_{l}}-p_{2}$, or $q_{2}-\sum_{l-1}^{q_{1}} x_{i_{l}}$. Suppose that we have an entry of the former type. Since $p_{2}+1>\sum_{l=1}^{p_{1}} x_{i_{l}}>p_{2}$, we find that $p_{2}=\left\lfloor\sum_{l=1}^{p_{1}} x_{i_{l}}\right\rfloor$. Observe that $p_{1}>\sum_{l=1}^{p_{1}} x_{i_{l}} \geq \sum_{i=n-p_{1}+1}^{n} x_{i}=n-1-\sum_{i=1}^{n-p_{1}} x_{i}>p_{1}-1$. Hence we find that $\left\lfloor\sum_{l=1}^{p_{1}} x_{i_{l}}\right\rfloor=p_{1}-1$, so that in fact our entry in $A$ is $\sum_{l=1}^{p_{1}} x_{i_{l}}-p_{1}+1$. A similar argument shows that for an entry of the form $q_{2}-\sum_{l=1}^{q_{1}} x_{i_{l}}$, we have $q_{2}=$ $q_{1}=\left\lceil\sum_{l=1}^{q_{1}} x_{i_{l}}\right\rceil$, so that the entry is given by $q_{1}-\sum_{l=1}^{q_{1}} x_{i_{l}}$.

Observe for any $q=1, \ldots, n$ we have $q-\sum_{l=1}^{q} x_{i_{l}}=\sum_{l=1}^{q}\left(1-x_{i_{1}}\right) \geq 1-x_{1}$. Similarly for any $p=1, \ldots, n-1$, we have $\sum_{l=1}^{p} x_{i_{l}}-p+1=1-\sum_{l=1}^{p}\left(1-x_{i_{l}}\right) \geq$ $1-\sum_{l=2}^{n}\left(1-x_{l}\right)=1-x_{1}$. Hence we find that each positive entry in $A$ is bounded below by $1-x_{1}=1-\max \left\{c_{1}-1, c_{2}\right\}=\min \left\{2-c_{1}, 1-c_{2}\right\}$.

Corollary 4.3. Suppose that $M$ is a product of $m$ matrices $A_{1}, \ldots, A_{m}$, each of which is an extreme point of $\mathcal{S}(c)$. Then the minimum positive entry in $M$ is bounded below by $\gamma^{m}$, where $\gamma=\min \left\{2-c_{1}, 1-c_{2}\right\}$.

Proof. Observe that any entry in $M$ consists of a sum of products of the form $\Pi_{j=1}^{m}\left(A_{j}\right)_{p_{j} q_{j}}$. If $M$ has a positive entry in some position, then at least one of the corresponding summands is positive, and the conclusion now follows readily from Lemma 4.2. $\square$

We now consider products of matrices that are extreme points in $\mathcal{S}(c)$.

LEMMA 4.4. Let $c$ be an admissible column sum vector of order $n$, with $2>c_{1}>$ $1, c_{j}<1, j=2, \ldots, n$. Set $k=n^{2}-3 n+3$, and suppose that $A_{1}, A_{2}, \ldots, A_{k}$ is a collection of matrices in $\mathcal{S}(c)$. Then the product $A_{1} \ldots A_{k}$ is a scrambling matrix. 
Proof. First, we note that it suffices to show that for any collection of extreme points $M_{1}, \ldots, M_{k}$ in $\mathcal{S}(c)$, the product $M_{1} \ldots M_{k}$ is a scrambling matrix. This follows from the fact that if we have any collection of matrices $A_{1}, A_{2}, \ldots, A_{k}$ in $\mathcal{S}(c)$, the product can be written as a convex combination of $k$-fold products of extreme points in $\mathcal{S}(c)$.

Next, we claim that if $M_{1}, \ldots, M_{k}$ are extreme points in $\mathcal{S}(c)$, then $M_{1} \ldots M_{k}$ is a scrambling matrix. To see the claim, suppose that we have the product $M_{q+1} \ldots M_{k}$ for some $q$ between 1 and $k-1$; evidently if $M_{q+1} \ldots M_{k}$ is a scrambling matrix, then so is $M_{1} \ldots M_{k}$. So, suppose that $M_{q+1} \ldots M_{k}$ is not a scrambling matrix. Let the bipartite graph of $M_{q}$ be $\mathcal{B}$. For each $j=1, \ldots, n$, let $\mathcal{R} i_{j}$ denote the row vertex of $\mathcal{B}$ such that the edge $\mathcal{R} i_{j} \sim \mathcal{C} j$ is in $\mathcal{B}$ 's perfect matching. For each $j=2, \ldots, n$, note that vertex $\mathcal{R} i_{j}$ has degree 2 in $\mathcal{B}$; for each such $j$, let $\mathcal{C} g(j)$ denote the other column vertex in $\mathcal{B}$ that is adjacent to $\mathcal{R} i_{j}$. Note also that $\mathcal{R} i_{1}$ is a pendant row vertex in $\mathcal{B}$.

We now consider the number of positive entries in $M_{q} \ldots M_{k}$. We claim that since $M_{q+1} \ldots M_{k}$ is not a scrambling matrix, the number of positive entries in $M_{q} \ldots M_{k}$ is strictly greater than that in $M_{q+1} \ldots M_{k}$. In order to help verify the claim, we introduce the following notation: given a nonnegative row vector $u^{T}$, we let $\mathbf{p}\left(u^{T}\right)$ denote the $(0,1)$ row vector of the same order that has zeros in the positions where $u^{T}$ is 0 , and ones in the positions where $u^{T}$ is positive.

To see the claim, first note that we have $e_{i_{1}}^{T} M_{q} \ldots M_{k}=e_{1}^{T} M_{q+1} \ldots M_{k}$; further for each $j=2, \ldots, n$, there is an $\alpha_{j} \in(0,1)$ such that

$$
e_{i_{j}}^{T} M_{q} \ldots M_{k}=\alpha_{j} e_{j}^{T} M_{q+1} \ldots M_{k}+\left(1-\alpha_{j}\right) e_{g(j)}^{T} M_{q+1} \ldots M_{k} .
$$

We thus find that the number of positive entries in $M_{q} \ldots M_{k}$ is at least as large as the number of positive entries in $M_{q+1} \ldots M_{k}$. Also, if for some index $j=2, \ldots, n$ the number of positive entries in $e_{i_{j}}^{T} M_{q} \ldots M_{k}$ coincides with the number of positive entries in $e_{j}^{T} M_{q+1} \ldots M_{k}$, then necessarily $\mathbf{p}\left(e_{g(j)}^{T} M_{q+1} \ldots M_{k}\right) \leq \mathbf{p}\left(e_{j}^{T} M_{q+1} \ldots M_{k}\right)$, where the inequality holds entrywise.

If $M_{q} \ldots M_{k}$ and $M_{q+1} \ldots M_{k}$ have the same number of positive entries, then it must be the case that for each $j=2, \ldots, n, \mathbf{p}\left(e_{g(j)}^{T} M_{q+1} \ldots M_{k}\right) \leq \mathbf{p}\left(e_{j}^{T} M_{q+1} \ldots M_{k}\right)$. Fix such an index $j$, and consider the unique path in $\mathcal{B}$ from vertex $\mathcal{C} j$ to vertex $\mathcal{C} 1$. That path has the form $\mathcal{C} j \sim \mathcal{R} i_{j} \sim \mathcal{C} g(j) \sim \mathcal{R} i_{g(j)} \sim \mathcal{C} g(g(j)) \sim \ldots \sim \mathcal{C} 1$. But then we have $\mathbf{p}\left(e_{j}^{T} M_{q+1} \ldots M_{k}\right) \geq \mathbf{p}\left(e_{(g(j))}^{T} M_{q+1} \ldots M_{k}\right) \geq \ldots \geq \mathbf{p}\left(e_{1}^{T} M_{q+1} \ldots M_{k}\right)$. Thus, if $M_{q} \ldots M_{k}$ and $M_{q+1} \ldots M_{k}$ have the same number of positive entries, then for each $j=1, \ldots, n$, the zero-nonzero pattern of $e_{1}^{T} M_{q+1} \ldots M_{k}$ is a subpattern of the zero-nonzero pattern of $e_{j}^{T} M_{q+1} \ldots M_{k}$. But then we find that $M_{q+1} \ldots M_{k}$ must have an all-positive column, contrary to our hypothesis that $M_{q+1} \ldots M_{k}$ is not a scrambling matrix. Consequently, the number of positive entries in $M_{q} \ldots M_{k}$ is strictly greater than that in $M_{q+1} \ldots M_{k}$, as claimed. 
Recall that $M_{k}$ has $2 n-1$ positive entries. Applying induction and the claim above, we find that for each $q=1, \ldots, k$, either $M_{q} \ldots M_{k}$ is a scrambling matrix, or it has at least $2 n-1+k-q$ positive entries. In particular, either $M_{1} \ldots M_{k}$ is a scrambling matrix, or it has at least $2 n+k-2=n^{2}-n+1$ positive entries. It is straightforward to show that any stochastic matrix with at least $n^{2}-n+1$ positive entries must be a scrambling matrix, so that in either case we find that $M_{1} \ldots M_{k}$ is scrambling, as desired.

Here is the main result of this section.

TheOREM 4.5. Suppose that $c^{T}$ is an admissible column sum vector of order $n$ and that $2>c_{1}>1, c_{j}<1, j=2, \ldots, n$. Let $\gamma=\min \left\{2-c_{1}, 1-c_{2}\right\}$. Then $\overline{\lambda_{2}(c)} \leq\left(1-\gamma^{n^{2}-3 n+3}\right)^{\frac{1}{n^{2}-3 n+3}}$.

Proof. Suppose that $A \in \mathcal{S}(c)$. Then there are extreme points $M_{1}, \ldots, M_{l}$ such that $A$ can be written as a convex combination $\sum_{j=1}^{l} \alpha_{j} M_{j}$, where $\alpha_{j}>0, j=1, \ldots, l$ and $\sum_{j=1}^{l} \alpha_{j}=1$. It follows that $A^{n^{2}-3 n+3}$ can be written as a convex combination of $\left(n^{2}-3 n+3\right)$-fold products of the matrices $M_{1}, \ldots, M_{l}$. Let $P$ denote such a product. By Lemma 4.4, $P$ is a scrambling matrix and from Corollary 4.3 , we find that each positive entry in $P$ is bounded below by $\gamma^{n^{2}-3 n+3}$. Consequently, we see that $\tau(P) \leq 1-\gamma^{n^{2}-3 n+3}$, and since $A^{n^{2}-3 n+3}$ is a convex combination of such products, we have $\tau\left(A^{n^{2}-3 n+3}\right) \leq 1-\gamma^{n^{2}-3 n+3}$. The conclusion follows by noting that $\left|\lambda_{2}(A)\right|=\left|\lambda_{2}\left(A^{n^{2}-3 n+3}\right)\right|^{\frac{1}{n^{2}-3 n+3}} \leq \tau\left(A^{n^{2}-3 n+3}\right)^{\frac{1}{n^{2}-3 n+3}}$. $\mathrm{B}$

The following example yields a lower bound on $\overline{\lambda_{2}(c)}$.

EXAMPLE 4.6. Suppose that $c^{T}$ is an admissible column sum vector of order $n \geq 3$, with $2>c_{1}>1, c_{j}<1, j=2, \ldots, n$. Consider the matrix

$$
A=\left[\begin{array}{ccc|c}
0 & c_{2} & 1-c_{2} & O^{T} \\
1 & 0 & 0 & O^{T} \\
c_{1}-1 & 0 & 2-c_{1} & O^{T} \\
\hline O & O & \left(\frac{c_{1}+c_{2}+c_{3}-3}{n-3}\right) \mathbf{1} & \frac{1}{n-3} \mathbf{1} u^{T}
\end{array}\right],
$$

where $u^{T}$ is the subvector of $c^{T}$ on its last $n-3$ columns. (In the case that $n=3$, we simply take $A$ to be the leading $3 \times 3$ principal submatrix above.) Then $A \in \mathcal{S}(c)$, and it is straightforward to determine that its eigenvalues are $1, \frac{u^{T} 1}{n-3}$ (if $n \geq 4$ ), 0 (of multiplicity $n-4$, again if $n \geq 4$ ) and

$$
\frac{1}{2}\left(-\left(c_{1}-1\right) \pm \sqrt{\left(c_{1}-1\right)^{2}+4 c_{2}\left(2-c_{1}\right)}\right) .
$$

In particular, $\left|\lambda_{2}(A)\right| \geq \frac{1}{2}\left(\left(c_{1}-1\right)+\sqrt{\left(c_{1}-1\right)^{2}+4 c_{2}\left(2-c_{1}\right)}\right)$. Since $c_{2} \geq \frac{n-c_{1}}{n-1}$, we find that $\left|\lambda_{2}(A)\right| \geq \frac{1}{2}\left(\left(c_{1}-1\right)+\sqrt{\left(3-c_{1}\right)^{2}+\frac{4}{n-1}\left(c_{1}-1\right)\left(c_{1}-2\right)}\right)$. 
A straightforward series of computations shows that the function

$$
\frac{1}{2}\left((x-1)+\sqrt{(3-x)^{2}+\frac{4}{n-1}(x-1)(x-2)}\right)
$$

is minimized on $x \in[1,2]$ at the point $x=\frac{3(n+1)-\sqrt{(2 n-3)(n-1)}}{n+3}$, and that the corresponding minimum value is $1-\frac{1}{n+3}\left(3-\frac{8 n+4-\frac{8}{n-1}}{\sqrt{(2 n-3)(n-1)}+\sqrt{2 n^{2}+11 n+11-\frac{16}{n-1}}}\right)$.

In particular, we see that

$$
\overline{\lambda_{2}(c)} \geq 1-\frac{1}{n+3}\left(3-\frac{8 n+4-\frac{8}{n-1}}{\sqrt{(2 n-3)(n-1)}+\sqrt{2 n^{2}+11 n+11-\frac{16}{n-1}}}\right) .
$$

Observe that this lower bound on $\overline{\lambda_{2}(c)}$ is asymptotic to $1-\frac{3-2 \sqrt{2}}{n}$ as $n \rightarrow \infty$.

As in Section 3, we have a weak ergodicity result for forward products of matrices in $\mathcal{S}(c)$.

THEOREM 4.7. Suppose that $c^{T}$ is an admissible column sum vector of order $n \geq 3$ with $2>c_{1}>1$ and $c_{j}<1, j=2, \ldots, n$. Let $A_{k}, k \in \mathbb{N}$ be a sequence of matrices in $\mathcal{S}(c)$. Then the sequence of forward products $T_{k}=A_{1} A_{2} \ldots A_{k}, k \in \mathbb{N}$, is weakly ergodic.

Proof. Set $r=n^{2}-3 n+3$ and let $\gamma=\min \left\{2-c_{1}, 1-c_{2}\right\}$. As in the proof of Theorem 4.5, we find that for any collection of extreme points $M_{1}, \ldots, M_{r}$ in $\mathcal{S}(c), \tau\left(M_{1} \ldots M_{r}\right) \leq\left(1-\gamma^{r}\right)$. We now find that for any collection of $r$ matrices $T_{1}, \ldots, T_{r} \in \mathcal{S}(c)$ we also have $\tau\left(T_{1} \ldots T_{r}\right) \leq\left(1-\gamma^{r}\right)$. Thus for each $k \in \mathbb{N}$, the forward product $A_{1} \ldots A_{k}$ satisfies $\tau\left(A_{1} \ldots A_{k}\right) \leq\left(1-\gamma^{r}\right)^{\left\lfloor\frac{k}{r}\right\rfloor}$. The conclusion now follows. $\square$

5. Open problems. In this section we pose a few open problems in the hopes that they will stimulate future research.

Problem 1: Let $c^{T}$ be an admissible column sum vector of order $n$ with $c_{1}>n-1$. Characterise the matrices $A \in \mathcal{S}(c)$ such that $\left|\lambda_{2}(A)\right|=\overline{\lambda_{2}(c)}=n-c_{1}$.

Problem 2: Improve the upper bounds on $\overline{\lambda_{2}(c)}$ given in Corollary 3.5 and Theorem 4.5 for Cases i) and ii), respectively.

Problem 3: Is it true that in Cases i) and ii), there is an extreme point $A$ of $\mathcal{S}(c)$ such that $\left|\lambda_{2}(A)\right|=\overline{\lambda_{2}(c)}$ ? If so, that would serve to narrow the class of matrices that 
need to be considered in addressing Problem 2 above.

Problem 4: Suppose that $c^{T}$ is an admissible column sum vector of order $n$, with $2>c_{1}>1$ and $c_{j}<1, j=2, \ldots, n$. The proof of Lemma 4.4 shows that any product of any $n^{2}-3 n+3$ matrices in $\mathcal{S}(c)$ must be a scrambling matrix. For each $n \geq 3$ find the minimum $k$ such that the product of any $k$ matrices in $\mathcal{S}(c)$ is a scrambling matrix. By considering the powers of the matrix

$$
\left[\begin{array}{cccccc}
1 & 0 & 0 & \cdots & 0 & 0 \\
\left(c_{1}-1\right) & \left(2-c_{1}\right) & 0 & 0 & \cdots & 0 \\
0 & \left(\sum_{j=1}^{2} c_{j}-2\right) & \left(3-\sum_{j=1}^{2} c_{j}\right) & 0 & \cdots & 0 \\
\vdots & & \ddots & \ddots & \ddots & \vdots \\
0 & 0 & \ldots & 0 & \left(\sum_{j=1}^{n-1} c_{j}-(n-1)\right) & c_{n}
\end{array}\right],
$$

we see that the minimum $k$ above is at least $n-1$.

Evidently it suffices to consider the case that the matrices under consideration are extreme points of $\mathcal{S}(c)$. Also, it is known (see [3]) that any product of $n-1$ fully indecomposable stochastic matrices of order $n$ must have all positive entries, so the problem may be restricted to the case that some of the matrices in the product fail to be fully indecomposable.

In addition to its inherent combinatorial interest, a solution to this problem would lead to an improved upper bound on $\overline{\lambda_{2}(c)}$ in Case ii).

Acknowledgment: The author is grateful to two anonymous referees, whose suggestions helped to improve the presentation of the results in this paper.

\section{REFERENCES}

[1] F. Bauer, E. Deutsch and J. Stoer. Abschätzungen für die Eigenwerte positiver linearer Operatoren. Linear Algebra and its Applications, 2:275-301, 1969.

[2] R. Dobrushin. Central limit theorem for nonstationary Markov chains I, II. Theory of Probability and Applications, 1:65-80, 329-383, 1956.

[3] D. Hartfiel. Nonhomogeneous Matrix Products. World Scientific, New Jersey, 2002.

[4] S. Kirkland. Girth and subdominant eigenvalues for stochastic matrices. Electronic Journal of Linear Algebra, 12:25-41, 2005.

[5] S. Kirkland. A cycle-based bound for subdominant eigenvalues of stochastic matrices. Linear and Multilinear Algebra, 57:247-266, 2009.

[6] R. Merris. Graph Theory. Wiley, New York, 2001.

[7] U. Paun. A class of ergodicity coefficients, and applications. Mathematical Reports (Bucuresti), $4(54): 225-232,2002$. 
[8] U. Rothblum and C.P. Tan. Upper bounds on the maximum modulus of subdominant eigenvalues of nonnegative matrices. Linear Algebra and its Applications, 66:45-86, 1985.

[9] E. Seneta. Non-negative Matrices and Markov Chains. Springer-Verlag, New York, 1981.

[10] V. Yemelichev, M Kovalev, and M. Kravtsov. Polytopes, graphs and optimisation. (Translated from Russian by G. Lawden.) Cambridge University Press, Cambridge, 1984 